\title{
La OTODI Lombardia: SLOTO
}

\author{
Gianni Randelli \\ Divisione di Ortopedia e Traumatologia, Ospedale San Donato, San Donato Milanese, Milano, Italia \\ gianni.randelli@grupposandonato.it
}

(c) Springer-Verlag Italia 2015

Nella storia ortopedica italiana l'OTODI (Ortopedici Traumatologi Ospedalieri d'Italia), ha rivestito un ruolo molto importante grazie al notevole bagaglio culturale dei propri soci, sin dalla seconda metà del secolo scorso. L'esposizione della notevole esperienza dei chirurghi ortopedici ospedalieri, nei congressi annuali OTODI, veniva apprezzata anche dai colleghi universitari i quali riconobbero presto l'OTODI come interlocutore affidabile e sincero.

L'Università dunque riconobbe l'importanza dell"Ospedale accettando che l'OTODI esprimesse un presidente ospedaliero della Società Italiana di Ortopedia e Traumatologia (SIOT) in alternanza, ogni due anni, con un presidente Universitario.

Queste battaglie vinte sul campo ebbero anche l'effetto di far quasi scomparire la storica animosità tra Ospedale e Università.

Agli inizi degli anni novanta dello scorso secolo l'OTODI entrava in una fase quasi letargica, per cui qualche importante socio, incominciava a suggerire lo scioglimento della Società. Fui assolutamente contrario a questa soluzione, rivendicando per l'OTODI la rappresentanza degli Ospedalieri, anche se non vi erano più attriti con il mondo Universitario.

Per la fortuna di OTODI, due illustri colleghi, il Prof. Aldo Maiotti ed il Prof. Galeazzo Carreri, avevano creato un OTODI Regionale del Lazio: l'ALOTO. Questa società era molto viva, aveva coinvolto tutti gli ortopedici ospedalieri laziali, sia da un punto di vista scientifico con manifestazioni congressuali locali che con viaggi di scambio culturale a livello delle più importanti realtà ospedaliere del globo.

In un viaggio a Siviglia, Maiotti, Carreri ed io, seduti nel bar dell'albergo dove eravamo ospiti, parlammo a lungo specialmente riguardo le mie perplessità ed il mio dispiacere di veder, forse, presto finire l'esperienza OTODI. Maiotti e Carreri, restarono esterrefatti e quasi mi assalirono, affermando che il futuro sarebbe stato nel creare Società Regionali OTODI come l'ALOTO, affiliate all'OTODI Nazionale.

In effetti politicamente in Italia, la Sanità era diventata appannaggio del controllo regionale e pertanto una Società ospedaliera regionale poteva essere importante quale controparte nella soluzione di problematiche sanitarie locali. Quella sera discutemmo a lungo e, alla fine decidemmo che l'OTODI nazionale non solo non doveva scomparire ma doveva diventare una federazione che raggruppasse varie OTODI regionali.

Andammo a dormire solo quando promisi che avrei creato in Lombardia una Società OTODI regionale; non solo, ma anche che avremmo insieme cercato altri proseliti in tutte le regioni italiane.

Il 6 ottobre 1995 io con altri 14 colleghi lombardi, fondammo a Milano la SLOTO: "Sodalizio Lombardo Ortopedici Traumatologi Ospedalieri”. Negli anni a seguire, prima la Campania e poi tutte le altre regioni seguirono questo esempio e fondarono delle OTODI regionali. L'OTODI nazionale oggi è una Federazione di Società regionali.

La SLOTO, oggi, è un Sodalizio molto vivo e dinamico soprattutto da un punto di vista culturale, con riunioni d'aggiornamento a livello provinciale e con un congresso regionale annuale.

La SLOTO si confronta inoltre con successo con il Governo Regionale Lombardo sulle principali problematiche che interessano la politica sanitaria specialistica.

Questo numero della rivista Lo Scalpello, affidato alla Lombardia, è dedicato alla chirurgia protesica articolare posttraumatica ed esprime il più alto livello culturale dell'Ortopedia Lombarda.

La SLOTO, partita nel 1995 con 15 soci, oggi annovera 1.000 soci.

Grazie a tutti coloro che mi hanno aiutato a vivere questo sogno. 